\title{
Variações espaço-temporais na abertura do dossel em diferentes tipologias vegetais sob efeito de fogo
}

\author{
Variations in canopy gaps in different phytophysiognomies under fire effect
}

\author{
Bruno Natali de Almeida', Rubens Manoel dos Santos ${ }^{\mathrm{II}}$, \\ Jean Daniel Morel ${ }^{\mathrm{III}}$, Diego Gualberto Salles Pereira' ${ }^{\mathrm{IV}}$
}

\begin{abstract}
Resumo
Os domínios fitogeográficos Atlântico e dos Cerrados brasileiros destacam-se pelo alto grau de ameaça de seus remanescentes, fazendo com que o estudo de sua ecologia seja de suma importância para a conservação desses domínios. Apesar da existência de distúrbios ecológicos naturais, a ação humana pode agravar a ocorrência desses fenômenos, como por exemplo: o aumento da frequência do fogo em ambientes naturais. Mesmo o dossel, camada superior da vegetação, é prejudicado direta ou indiretamente pela ação desse distúrbio. Além disso, as características do dossel são determinantes para a dinâmica de regeneração das comunidades vegetais, pois suas condições regulam os níveis de luminosidade que chegam às camadas inferiores da vegetação. Assim, este trabalho buscou estudar as variações na abertura do dossel de um mosaico vegetacional inserido em uma área de contato entre os domínios Atlântico e dos Cerrados, com o intuito de identificar como se dão essas alterações entre as tipologias vegetais analisadas e qual a influência da ocorrência de fogo nas oscilações no estrato superior da vegetação. Para isso, o estudo acompanhou as mudanças na abertura do dossel, com o uso de fotografias hemisféricas, em diferentes unidades vegetacionais por três anos, a partir da ocorrência de um evento de fogo no local. Os resultados obtidos mostraram que fitofisionomias semelhantes apresentaram níveis de abertura do dossel parecidos, da mesma forma que exibiram um padrão de oscilação similar entre si. Por fim, depreende-se que o fogo se mostrou como um modificador indireto do dossel, uma vez que as próprias características da vegetação têm a capacidade de controlar os impactos desse tipo de distúrbio.
\end{abstract}

Palavras-chave: Luminosidade; Distúrbios ecológicos; Fotografia hemisférica; Vegetação

\begin{abstract}
Atlantic and Brazilian savanna phytogeographic domains stand out the high level of threating from forest remnants, making the ecological study of these important areas for both the Domains conservation. In nature, disturbances events are common, but human activities can aggravate the occurrence of these phenomena, for example, increasing the frequency of fire events in natural environments. Even the higher layer in the forest, the canopy, suffers directly and indirectly affected by this disturbance. Besides, canopies characteristics are decisive factor acting in the regeneration dynamics of vegetal community, regulating luminosity that reach inferior low layers of vegetation. This study aims to capture the variations in canopy gaps in an ecotone area between Savanna and Atlantic Domains in order to identify how these changes occur between the analyzed vegetation types and which is the fire influence in the upper layer of the forest. We captured variations in the forest gaps after a fire event utilizing hemispheric photos. The photos have been taking for three years in an interval of six months. We found out that similar phytophysiognomies showed similar levels of canopy coverage and displayed an oscillation pattern. Finally, it appears that fire proved to be an indirect modifier of the canopy, since the characteristics of the vegetation itself have the ability to control the impacts of this type of disturbance.
\end{abstract}

Keywords: Luminosity; Ecological disturbances; Hemispheric photo; Vegetation

I Biólogo, Me., Professor do Curso de Biologia, Centro Universitário Teresa D’Ávila, Av. Dr. Peixoto de Castro, 539 - Vila Celeste, CEP 12606-580, Lorena (SP), Brasil. brunonatali1987@ hotmail.com (ORCID: 0000-0002-4217-6704)

II Biólogo, Dr., Professor, Departamento de Ciências Florestais, Universidade Federal de Lavras, Caixa Postal 3037, CEP 37200-900, Lavras (MG), Brasil. rubensmanoel@ufla.br (ORCID: 0000-0002-4075-462X)

III Engenheiro Florestal / Geógrafo, Dr., Pesquisador, Departamento de Ciências Florestais, Universidade Federal de Lavras, Caixa Postal 3037, CEP 37200-900, Lavras (MG), Brasil. jeanmorel.ufla@gmail.com (ORCID: 0000-0002-6226-4779)

IV Biólogo, Me., Pesquisador, Departamento de Ciências Florestais, Universidade Federal de Lavras, Caixa Postal 3037, CEP 37200-900, Lavras (MG), Brasil. In memoriam 


\section{Introdução}

Atualmente, o crescimento da população humana tem ameaçado cada vez mais os ambientes naturais, comprometendo a biodiversidade e os serviços ambientais dos remanescentes de vegetação (BRASIL, 2013). No Brasil, destacam-se os domínios fitogeográficos Atlântico e dos Cerrados pelo alto risco à sua conservação, sendo considerados como hotspots para conservação da biodiversidade (MYERS et al., 2000).

O domínio Atlântico encontra-se extremamente fragmentado, com apenas cerca de $8 \%$ de sua cobertura original (GALINDO-LEAL; CÂMARA, 2005), contendo poucas conexões entre os remanescentes existentes, o que gera extinção de diversas espécies e a limitação da área de ocorrência de outras mais (TABARELLI et al., 2010; JOLY; METZEGER; TABARELLI, 2014). Assim como o Domínio Atlântico, os Cerrados têm sido fortemente afetados pelo desenvolvimento de atividades humanas, com expansão da fronteira agrícola e com o aumento da frequência do fogo (KLINK; MACHADO, 2005; BRASIL, 2013), culminando em uma redução de cerca de $80 \%$ da cobertura original do domínio (MYERS et al., 2000).

Diante da preocupação com o futuro desses domínios e do aumento de atividades humanas como o desmatamento e a fragmentação de habitats, pesquisas voltadas à recuperação desses ambientes tornam-se imperativas. A cobertura vegetal está em contato direto com a atmosfera, o que faz do dossel um importante regulador dos níveis de energia que incidem sobre a vegetação e sobre o solo (SANTOS et al., 2011). Assim, o dossel surge como um componente a ser estudado para melhoria das ações de regeneração da vegetação, uma vez que as características do dossel determinam as condições do sub-bosque, e, portanto, da dinâmica de regeneração das espécies (SOUZA; GANDOLFI; RODRIGUES, 2014), pois este é capaz de controlar a luminosidade e o microclima dos estratos inferiores da vegetação (BIANCHINI; PIMENTA; SANTOS, 2001; SANTOS et al., 2011).

Há distúrbios ecológicos que podem gerar danos na estrutura do dossel e, consequentemente, na dinâmica de regeneração de espécies. Dentre esses distúrbios ecológicos, destaca-se o fogo, um distúrbio de ocorrência natural, que está se tornando mais frequente devido à ação exploratória do homem (BOWMAN et al., 2009). O fogo é um importante agente modificador de ambientes (COCHRANE; SCHULZE, 1999) cujos impactos se estendem pelos diferentes compartimentos da vegetação, desencadeando uma substituição de espécies vegetais por meio de processos sucessionais (COCHRANE, 2003). Em queimadas de maior intensidade, o fogo pode chegar ao dossel, alterando diretamente as condições microclimáticas dos ambientes afetados, devido principalmente às mudanças na radiação solar sobre o sub-bosque (HENNENBERG et al., 2006; HOFFMANN et al.,2012).

Trabalhos de pesquisa que visem o estudo dos fatores que modificam o dossel e a luminosidade do sub-bosque são pouco comuns na literatura e os que existem, em geral, seguem as avaliações por um curto período de tempo, como em Rich et al. (1993), Nicotra, Chazdon e Iriarte (1999) e Bianchini, Pimenta e Santos (2001), que fizeram estudos por períodos próximos de um ano. Diante desse cenário, pesquisas que busquem o maior entendimento dos processos ecológicos e dos impactos da ação humana sobre os dosséis dos hotspots brasileiros, por meio de avaliações mais duradouras, são essenciais para a conservação dos seus remanescentes.

Assim, o presente trabalho busca prover informações a respeito do comportamento do dossel entre as diferentes unidades de vegetação de um fragmento florestal, por meio do acompanhamento dos valores de abertura do dossel ao longo de três anos. Esse fragmento está inserido em uma região de contato dos Domínios Atlântico e dos Cerrados, cuja maioria das unidades foi atingida por um evento de fogo, o que confere valor ecológico à região.

Nesse sentido, a presente pesquisa parte dos seguintes questionamentos: como se dá o comportamento do dossel ao longo do tempo após distúrbio? Esse comportamento é o mesmo entre diferentes formações vegetacionais? De acordo com os questionamentos expostos, tem-se a seguinte hipótese: (i) fitofisionomias com estrutura vegetacional e porte distintos apresentariam 
diferentes níveis de abertura do dossel, porém, exibindo uma oscilação semelhante ao longo do tempo.

\section{Material e método}

\section{Área de Estudo e Distribuição dos Pontos Amostrais}

O trabalho foi desenvolvido no Parque Ecológico Quedas do Rio Bonito (PEQRB), localizado no município de Lavras, MG, nas coordenadas geográficas $21^{\circ} 19^{\prime} \mathrm{S}$ e $44^{\circ} 59^{\circ} \mathrm{O}$. O clima da região é do tipo Cwb, segundo o mapa da classificação de Köppen para o Brasil, proposto por Alvares et al. (2013), contendo verões brandos e invernos secos. A precipitação anual média é de cerca de $1500 \mathrm{~mm}$, com temperatura anual média de $19,6^{\circ} \mathrm{C}$ (BRASIL, 1992). A Figura 1 exibe o climograma para os anos de 2010 a 2014, com dados oriundos da estação meteorológica de Lavras (a mais próxima da área de estudo).

Figura 1 - Climograma do Município de Lavras - MG, para o período de janeiro de 2010 a janeiro de 2015

Figure 1 - Weather graphics in Lavras - MG munincipality due to the months of January of 2010 and January of 2015

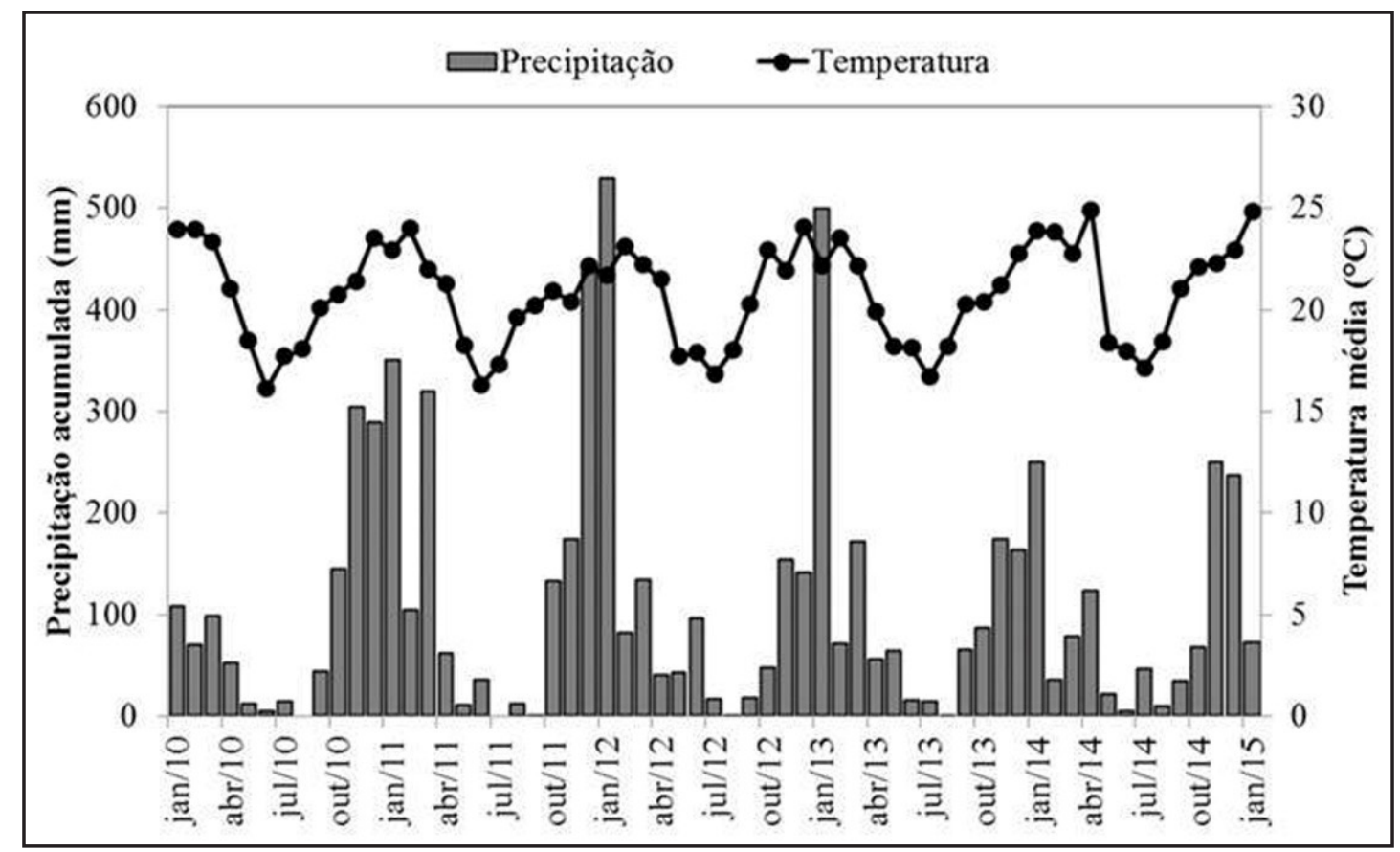

Fonte: Estação Meteorológica de Lavras (2016)

A área de estudo é o maior fragmento de vegetação nativa da região, com aproximadamente 200 ha, inserida no domínio Atlântico, porém com ocorrência de manchas de vegetação dos Cerrados, contendo formações de florestas estacionais semideciduais e Cerrado strictu senso (OLIVEIRA-FILHO; FLUMINHAN-FILHO, 1999). O local possui grande variação ambiental, com relevo acidentado, apresentando cotas altitudinais entre 1000 e $1300 \mathrm{~m}$, resultando em diversas formações vegetais sobre diferentes profundidades de solo, desde fisionomias campestres a ambientes de floresta alta e densa nos fundos de vale.

Entre o final de setembro e o início de outubro de 2011, houve um evento de fogo que 
atingiu parte da superfície da área de estudo. O fogo iniciou em áreas adjacentes ao parque, partindo de formações campestres e seguindo até os ambientes florestais, afetando diferentes fitofisionomias do PEQRB. Assim, considerando as variações fitofisionômicas e ambientais da área de estudo e a ocorrência do fogo, para esta pesquisa foram analisadas sete formações vegetacionais distintas (Tabela 1), abrangidas por 66 pontos de amostragem distribuídos pela área do parque, de acordo com a área de cobertura de cada formação (Figura 2).

\section{Tabela 1 - Descrição das Unidades de Vegetação Analisadas no Parque Ecológico Quedas} do Rio Bonito, Lavras, MG

Table 1 - Description of the Vegetation Units analyzed in Quedas do Rio Bonito Ecological Park, Lavras, MG

\begin{tabular}{|c|c|}
\hline Vegetação & Características \\
\hline Floresta não queimada (Fnq) & $\begin{array}{l}\text { Vegetação florestal, com dossel em torno de } 20 \mathrm{~m} \text { de altura, em uma encosta } \\
\text { (14 pontos amostrais). }\end{array}$ \\
\hline Floresta queimada $(\mathbf{F q})$ & $\begin{array}{l}\text { Vegetação florestal, contígua à floresta não queimada. Dossel em torno de } 20 \\
\mathrm{~m} \text { de altura, com crestamento do dossel em porções mais baixas, em área de } \\
\text { encosta (13 pontos amostrais). }\end{array}$ \\
\hline Candeal $(\mathbf{C d})$ & $\begin{array}{l}\text { Vegetação dominada por candeias (Eremanthus spp.), com dossel em torno de } \\
10 \mathrm{~m} \text {, com crestamento do dossel, em transição abrupta para as formações } \\
\text { campestres ( } 11 \text { pontos amostrais). }\end{array}$ \\
\hline Mata ciliar (Mc) & $\begin{array}{l}\text { Vegetação próxima a um curso d'água, afetada pelo fogo, contendo dois } \\
\text { pontos de amostragem próximos à margem do córrego que corta o parque e } \\
\text { outros três pontos seguindo um gradiente de elevação a partir do córrego ( } 5 \\
\text { pontos amostrais). }\end{array}$ \\
\hline Ecótono $(\mathrm{Ec})$ & $\begin{array}{l}\text { Vegetação de transição entre as formações florestais e campestres, sob } \\
\text { efeito do fogo. Os pontos amostrais estão distribuídos em quatro conjuntos } \\
\text { de três pontos. Os conjuntos de pontos compreendem uma amostragem em } \\
\text { área de floresta, um ponto em meio à transição floresta-campo e o terceiro } \\
\text { ponto em ambiente de campo (12 pontos amostrais). }\end{array}$ \\
\hline Pteridium sp. $(\mathrm{Pt})$ & $\begin{array}{l}\text { Vegetação de encosta com árvores espaçadas, que antes da passagem do fogo } \\
\text { era dominada por Pteridium sp. e após o incêndio passou a ser dominada por } \\
\text { gramíneas com cerca de } 1,5 \mathrm{~m} \text { de altura (6 pontos amostrais). }\end{array}$ \\
\hline Campo $(\mathrm{Cp})$ & $\begin{array}{l}\text { Vegetação nativa predominantemente herbácea, com árvores espaçadas e de } \\
\text { pequeno porte, sob efeito do fogo ( } 5 \text { pontos amostrais). }\end{array}$ \\
\hline
\end{tabular}

Fonte: Autores (2016) 
Figura 2 - Mapa do Parque Ecológico Quedas do Rio Bonito, localizado em Lavras, MG. O mapa apresenta a distribuição das fitofisionomias do parque e a locação dos pontos amostrais

Figure 2 - Quedas do Rio Bonito Ecological Park's map, located in Lavras, MG. The map shows the distribution of the phytophysiognomies of the park and the location of the sampling points

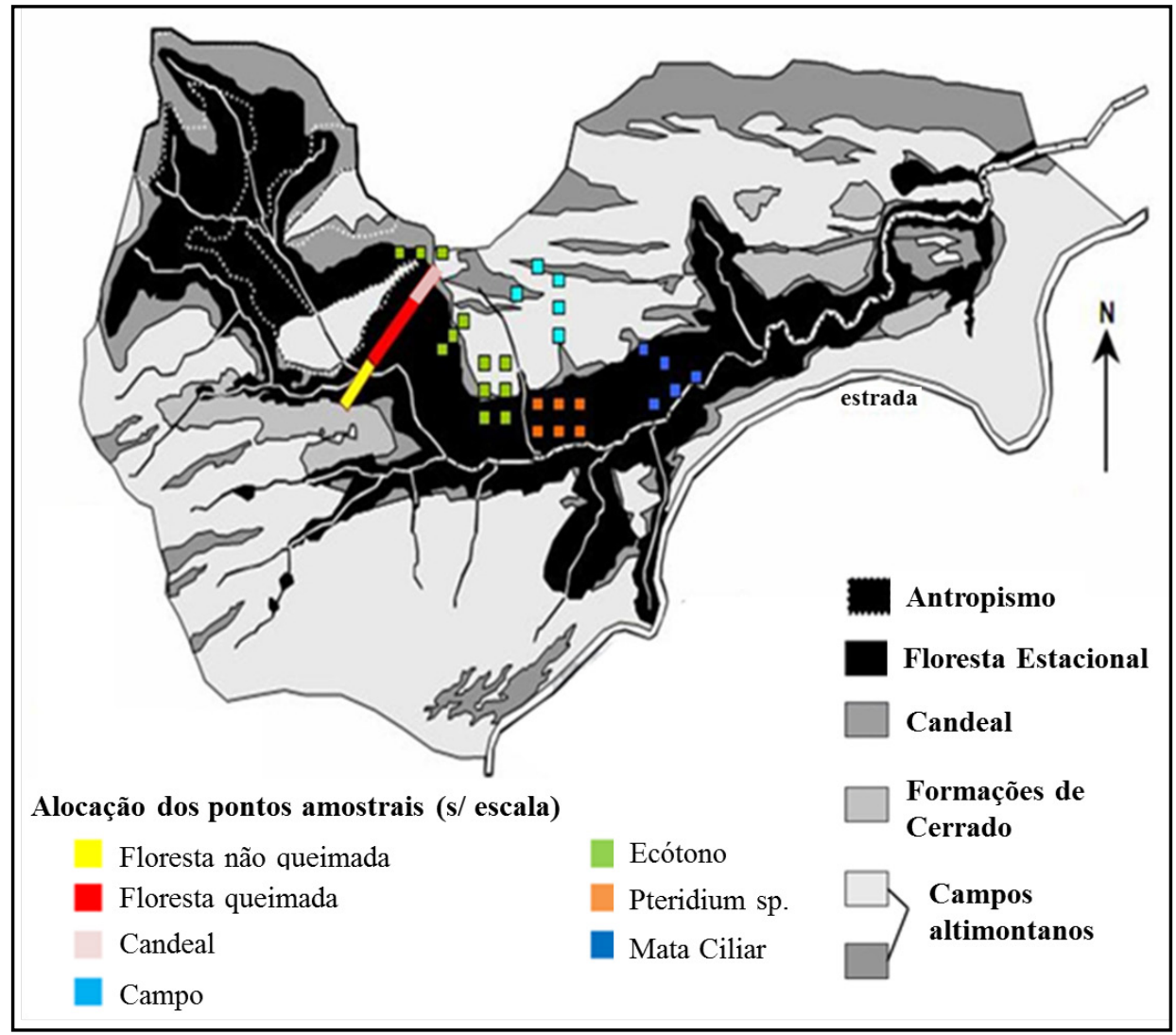

Fonte: Adaptado de Oliveira-Filho e Fluminhan-Filho (1999)

\section{Aquisição e Processamento das Imagens}

As imagens utilizadas neste estudo foram registradas desde o momento seguinte ao fogo (outubro de 2011), até o mês de outubro de 2014, em intervalos de seis meses, resultando em sete repetições, totalizando três anos de acompanhamento da vegetação. O intervalo entre as repetições deu-se de acordo com a estacionalidade climática da região, com fotografias obtidas em outubro, visando à amostragem ao fim da estação seca e fotografias em abril para amostragem ao fim da estação chuvosa, com o intuito de identificar alterações na vegetação relacionadas à deciduidade natural. Apenas na repetição referente à estação seca de 2013, a fotografia foi registrada em dezembro, ao invés de outubro, devido a reparos necessários na câmera.

As fotografias hemisféricas foram obtidas com uma câmera digital Nikon D90 de 12 megapixels, com acoplamento de uma lente objetiva convexa de $10,5 \mathrm{~mm}$ (conhecida como "lente olho de peixe"), apoiada em um tripé, mantendo a estabilidade da câmera com a lente a $1 \mathrm{~m}$ do solo. As fotos foram registradas a partir dos mesmos pontos de referência em todas as repetições, garantindo a homogeneidade do registro da imagem.

Buscando a minimização dos efeitos de luminosidade excessiva ou insuficiente e a eliminação de possíveis erros no momento da obtenção das imagens, para cada ponto fotografado, foram obtidas de 3 a 5 imagens com diferentes níveis de exposição à luz, de acordo com as 
condições do ambiente, como intensidade luminosa e ventos na ocasião do registro das imagens. Após a extração dos dados, tomou-se para as análises a média do conjunto de valores obtidos em cada fotografia por ponto e por repetição.

O processamento das imagens consistiu no processo de binarização (transformação dos pixels da imagem em branco ou preto) das fotografias registradas, realizada por meio do software ImageJ (ABRAMOFF; MAGALHÃES; RAM, 2004), e posterior extração dos dados de abertura do dossel com o software Gap Light Analyzer (FRAZER; CANHAM; LERTZMAN, 1999).

\section{Análise e Exibição dos Resultados}

Foi utilizada a abertura do dossel (AD) para avaliar as modificações das características do dossel dos diferentes ambientes analisados. A AD é uma variável apresentada em porcentagem e indica o quanto o dossel está aberto, permitindo inferir a respeito dos níveis de luminosidade do sub-bosque (MAURO-DIAZ; LENCINAS; DEL-VALLE, 2014). A porcentagem de abertura é calculada pela contagem de pixels da imagem classificados como céu (cor branca), após o processo de binarização (MAURO-DIAZ; LENCINAS; DEL-VALLE, 2014).

$\mathrm{Na}$ busca por evidenciar a distribuição dos níveis de AD em cada unidade vegetacional, confeccionou-se boxplots para cada unidade, exibindo o comportamento da AD ao longo do tempo. O boxplot é um tipo de gráfico que exibe a distribuição dos dados por meio de seus quartis e da mediana (nas caixas estão representados $50 \%$ dos dados observados), permitindo a comparação entre conjuntos de dados relativos às variáveis categóricas.

Já o comportamento do dossel frente à estacionalidade climática foi analisado por meio de gráficos com os dados de AD média para as repetições nas estações seca e úmida, em cada unidade amostral. Por fim, com o intuito de analisar a variação espacial entre as unidades de vegetação, foi construído um gráfico com os valores médios de $\mathrm{AD}$ de cada unidade ao longo das repetições. Dada a não normalidade na distribuição dos dados, verificada pelo teste Shapiro Wilk, as comparações foram submetidas ao Teste de Kruskall-Wallis para verificação estatística de similaridades na AD, seguido do Teste de Dunn para identificação dos grupos similares entre si, ambos testes a $5 \%$ de significância.

Neste trabalho, para realização das análises estatísticas e confecção de gráficos e tabelas foram usados os softwares R estatística (R CORE TEAM, 2014) e Microsoft Excel (MICROSOFT, 2010).

\section{Resultados e discussão}

A Figura 3 exibe os diagramas tipo boxplot para cada uma das unidades de vegetação amostradas. De acordo com o gráfico, a unidade Floresta não queimada (Fnq) apresentou oscilações em que os valores para outubro tendem a apresentar maior AD que os resultados para abril. Dessa forma, a 1 ${ }^{\mathbf{a}}$ e a $3^{\mathrm{a}}$ repetição apresentaram distribuição semelhante $(\mathrm{p}=0,4709)$, assim como a $2^{\underline{a}}, 4^{\underline{a}}, 5^{\underline{a}}$ e $6^{\underline{a}}$ repetição temporal que também se agruparam com menores valores, e a $7^{\underline{a}}$ repetição destacou-se das demais com valores de AD significativamente maiores.

Assim como em Fnq, a unidade Floresta queimada (Fq) apresentou oscilações na AD nas repetições de outubro e abril, porém em $\mathrm{Fq}$ as repetições foram mais semelhantes entre si, destacando-se a $7^{\underline{a}}$ repetição com maiores valores de $A D$ e estatisticamente iguais à $1^{\underline{a}}$ e $3^{\underline{a}}$ repetições, e a $5^{\underline{a}}$ repetição igual a todas outras com exceção da $7^{\mathfrak{a}}$ repetição.

Em Cd a $1^{\underline{a}}$ e a $7^{\underline{a}}$ repetição se destacaram com maior $\mathrm{AD}$, enquanto a $2^{\underline{a}}, 4^{\underline{a}}, 5^{\underline{a}}$ e a $6^{\underline{a}}$ repetição foram semelhantes aos menores valores de abertura. Mc não apresentou diferenças significativas entre as repetições (Kruskal-Wallis, $\mathrm{p}=0,44$ ), porém a 1르 e a $7^{\underline{a}}$ repetição apresentaram diferenças marginalmente significativas $(\mathrm{p}<0,07)$ se comparadas às $2^{\underline{a}}$ e $5^{\underline{a}}$ repetições. Já a unidade $\mathrm{Cp}$ manteve sua $\mathrm{AD}$ praticamente constante ao longo das repetições, sem diferenças estatísticas no tempo amostrado (Kruskal-Wallis, $\mathrm{p}=0,98$ ). 
Figura 3 - Boxplot da Abertura do Dossel das Unidades Amostrais Analisadas no Parque Ecológico Quedas do Rio Bonito entre as Repetições Temporais. Asteriscos representam outliers e os traços centrais das caixas indicam as medianas

Figure 3 - Boxplot showing the canopy gaps in the vegetation units analyzed at Quedas do Rio Bonito Ecological Park between seazonal repetitions. Asterisks represent outliers and central lines in the box represent median values

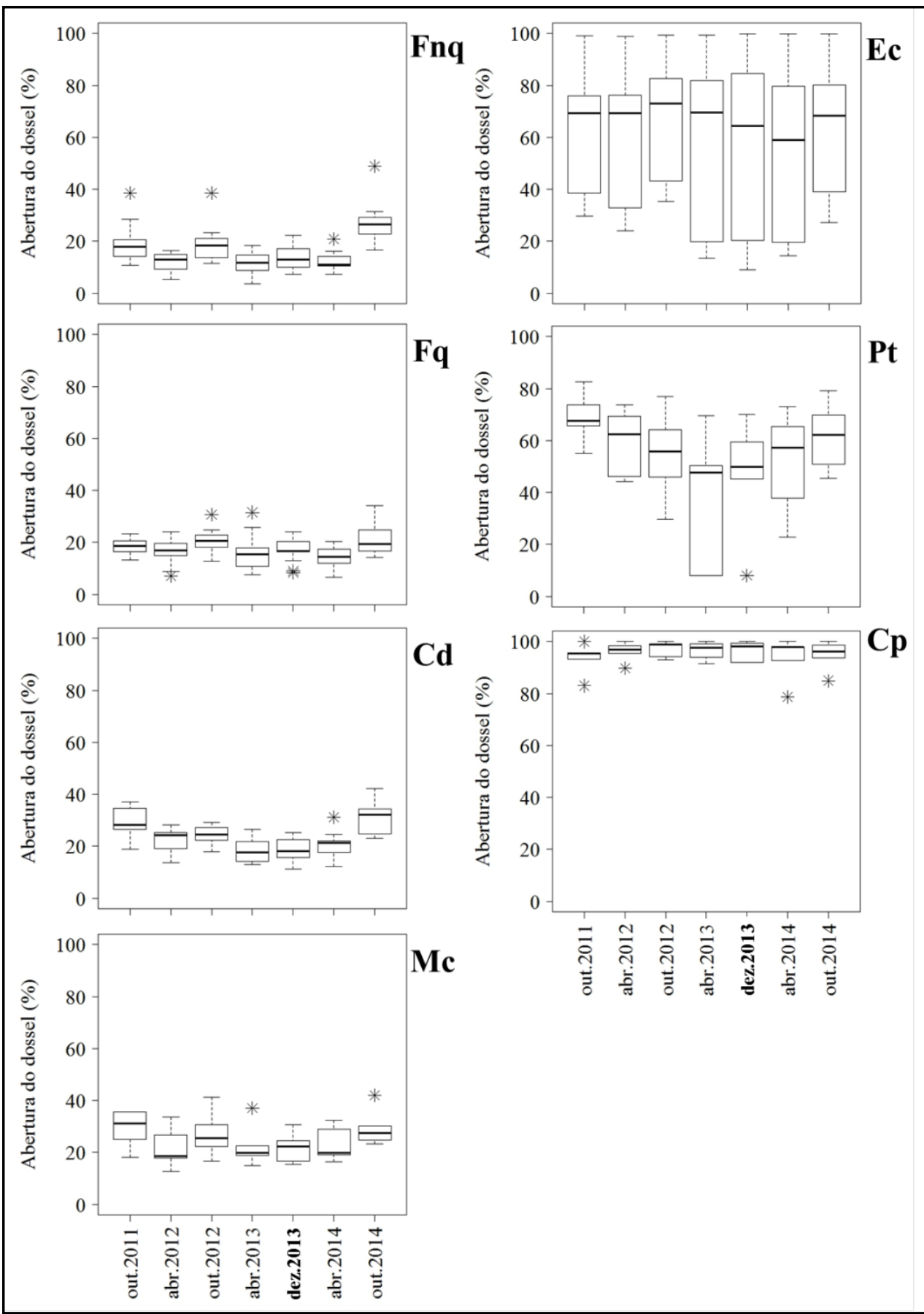

Fonte: Autores (2016)

Em que: $\mathrm{Fnq}=$ Floresta não queimada $; \mathrm{Fq}=$ Floresta queimada $; \mathrm{Cd}=$ Candeal; $\mathrm{Mc}=$ Mata ciliar Ec = Ecótono $\mathrm{Pt}=$ Pteridium $; \mathrm{Cp}=$ Campo. 
De acordo com os resultados exibidos na Figura 3, as unidades Fnq, Fq, Cd, Mc e Cp apresentaram baixa amplitude na variação da $\mathrm{AD}$ ao longo das repetições, $\mathrm{Cp}$ por não apresentar uma cobertura de dossel formada e as demais unidades por serem fisionomias florestais. A pequena variação da $\mathrm{AD}$ em $\mathrm{Cp}$ deve-se ao fato de existirem poucas árvores nessa unidade, sem a formação de um dossel contínuo, impossibilitando assim a captação de variações de AD ao longo do tempo. Nessa unidade, as pequenas alterações na abertura se deram pelo crescimento de indivíduos regenerantes e por inflorescências de espécies herbáceas próximas ao ponto de registro das imagens.

Os ambientes florestais, em geral, possuem dossel contínuo, permitindo que as oscilações na abertura sejam mais facilmente detectadas (NICOTRA; CHAZDON; IRIARTE, 1999; BIANCHINI; PIMENTA; SANTOS, 2001). As unidades Fnq, Fq, Cd e Mc exibiram as oscilações mais claras ao longo das repetições, de forma que, no presente estudo, essas fisionomias apresentaram uma tendência de maiores valores de AD na estação seca.

Essa condição está relacionada à estacionalidade climática da região de estudo, apresentando menor precipitação entre os meses mais frios (Figura 1). A deciduidade gera um aumento na luminosidade do sub-bosque devido aos mosaicos de AD nas unidades de fisionomia florestal, como relatado nos trabalhos de Bianchini, Pimenta e Santos (2001), Gandolfi, Joly e Rodrigues (2007) e Gandolfi, Joly e Leitão-Filho (2009), bem como verificado pelas análises da sazonalidade na AD para as unidades Fnq, Fq e Cd.

A unidade Mc, ao contrário do esperado por ser em uma tipologia florestal, não mostrou diferenças significativas entre as repetições e entre as estações, apesar da tendência de maior AD nos meses com menor precipitação. Essa unidade possui maior diversificação ambiental entre os pontos amostrais e é possível que a maior umidade próxima ao córrego tenha influenciado a AD nos pontos próximos ao rio, amenizando os efeitos da estação seca (NICOTRA; CHAZDON; IRIARTE, 1999; TEIXEIRA; ASSIS, 2009). Além disso, devido ao porte mediano desta formação, é provável que o dossel tenha sofrido impactos diretos do fogo, devido ao crestamento da copa observado nos primeiros registros das fotografias, o que também pode ter alterado a deciduidade.

$\mathrm{O}$ Ecótono $(\mathrm{Ec})$ demonstrou a maior amplitude de variação dos percentuais de $\mathrm{AD}$, sem diferenças estatisticamente significativas ao longo das repetições (Kruskal-Wallis, $\mathrm{p}=0,91$ ). A unidade Pteridium (Pt) também apresentou grande amplitude de variação na AD entre as repetições, com um comportamento diferenciado das demais unidades, mostrando menores níveis de $\mathrm{AD}$ na $4^{\mathrm{a}}$ medição (média de $38,50 \%$ ), com maiores valores na $1^{\underline{a}}$ e $7^{\mathrm{a}}$ medições (médias de 68,59 e $61,57 \%)$, de forma que a $1^{\underline{a}}$ repetição diferenciou-se estatisticamente da $4^{\underline{a}}$, $5^{\underline{a}}$ e $6^{\underline{a}}$ medição e a $7^{\text {a }}$ repetição diferenciou-se da $4^{\mathbf{a}}$.

Diante dos resultados, não foi identificado um padrão de oscilação da $A D$ para as unidades Ec e Pt. Supõe-se que isso esteja relacionado ao caráter transicional da unidade Ec e ao desenvolvimento de gramíneas em Pt. Em Ec, os pontos amostrais encontram-se tanto em fisionomias florestais como em áreas de campo, tornando difícil a percepção de um padrão na oscilação da AD, pois apesar de florestas evidenciarem oscilações características da sazonalidade na disponibilidade hídrica (HAINES; FOSTER, 1977; FENNER, 1998; MORELLATO et al., 2000; SINGH; KUSHWAHA, 2005), em campos já não é possível avaliar essa variação na AD, pois essas formações não apresentam dossel contínuo.

$\mathrm{Na}$ unidade $\mathrm{Pt}$, houve um desenvolvimento massivo de gramíneas, que cresceram além do ponto de registro das imagens, fazendo com que a maioria das fotografias tenha registrado a cobertura de gramíneas. Com isso, o comportamento da AD nessa unidade reflete as oscilações do crescimento de indivíduos da família Poaceae. Sendo assim, como as gramíneas não apresentam regime de deciduidade (JUDD et al., 2009), não foi encontrado um padrão de variação ligado à sazonalidade. Porém, devido à capacidade de regeneração após o fogo (BOND, 2008; SIMON et al., 2009) e o caráter colonizador desse grupo de plantas (JUDD et al., 2009), a variação na AD deste ambiente pode estar relacionada às mudanças no adensamento de gramíneas no local.

A Figura 4 reúne as oscilações da $\mathrm{AD}$ das sete unidades analisadas em uma mesma 
escala gráfica. De acordo com a figura, as unidades vegetacionais do parque formaram três agrupamentos semelhantes entre si: com as unidades Fnq, Fq, Cd e Mc variando entre 10 e $30 \%$ de AD; Ec e Pt entre 40 e 70\%; e a unidade Cp isolada das demais, com AD em torno de 90\%.

\section{Figura 4 - Abertura do Dossel entre as Unidades de Vegetação do Parque Ecológico Quedas do Rio Bonito. A figura exibe os valores médios e erro-padrão entre as unidades no decorrer das repetições temporais}

Figure 4 - Canopy Gaps in the Vegetation Unitsat Quedas do Rio Bonito Ecological Park. The figure shows medium values and standard error among the units during seazonal repetitions

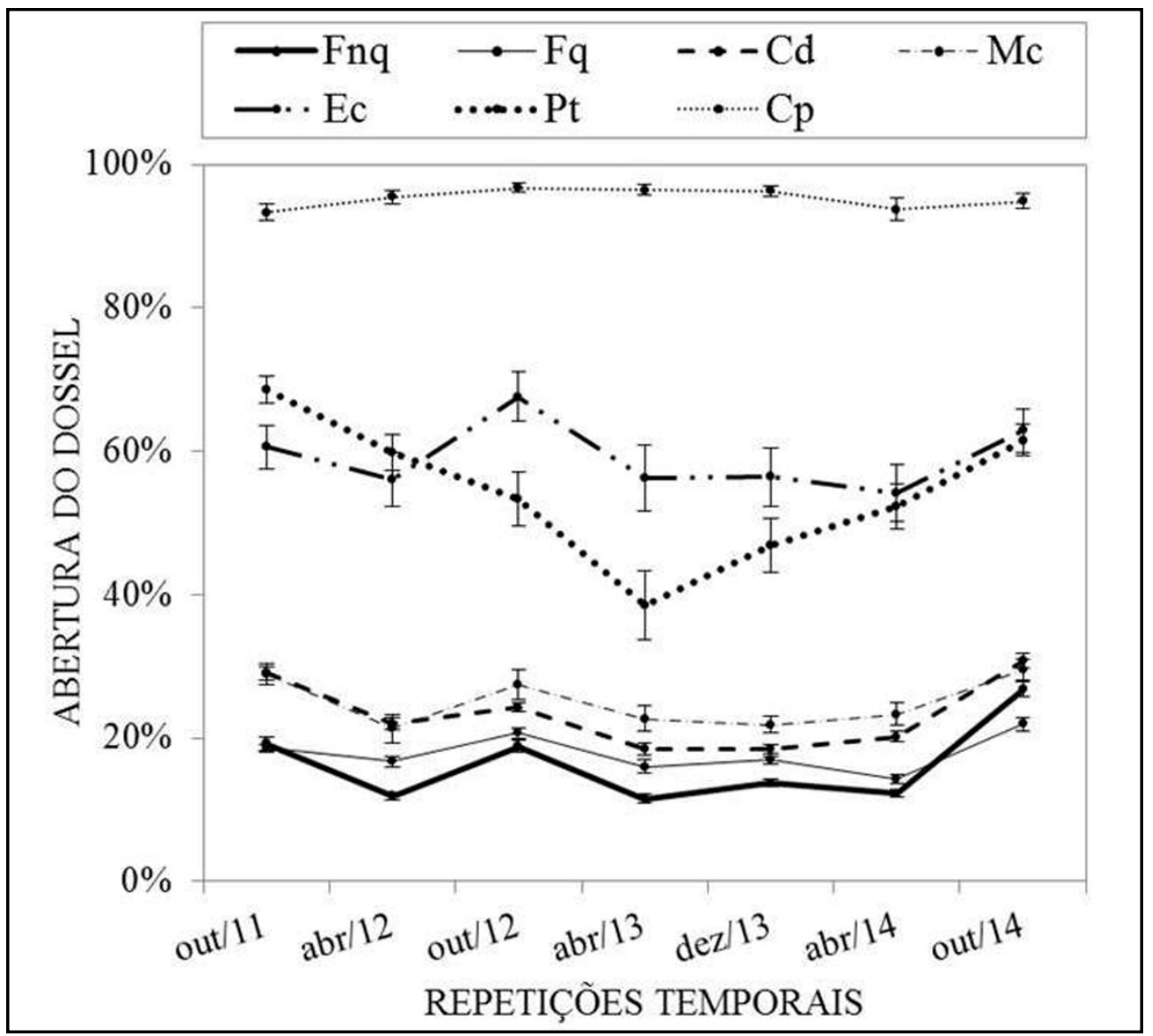

Fonte: Autores (2016)

Em que: Fnq = Floresta não queimada $; \mathrm{Fq}=$ Floresta queimada $; \mathrm{Cd}=$ Candeal $; \mathrm{Mc}=\mathrm{Mata}$ ciliar; Ec = Ecótono; $\mathrm{Pt}=$ Pteridium $; \mathrm{Cp}=$ Campo.

As unidades $\mathrm{Cd}$ e Mc são mais heterogêneas que Fnq e Fq, com Cd configurando um ambiente transicional contíguo às formações campestres do parque e Mc contendo pontos amostrais com maior variação ambiental entre si. Comparando-se os possíveis efeitos do fogo em Fnq e $\mathrm{Fq}$ com $\mathrm{Cd}$ e $\mathrm{Mc}$, acredita-se que $\mathrm{Cd}$ e $\mathrm{Mc}$ tenham sido mais afetadas pela queimada, haja vista apresentarem maior AD. Essa maior AD deve-se aos seus dosséis menos densos, mais baixos e heterogêneos do que as demais unidades de formações florestais, o que tornaria esses ambientes mais suscetíveis ao fogo pela maior entrada de luz no sub-bosque que levaria ao maior ressecamento do material combustível, favorecendo a propagação das chamas (COCHRANE et al., 1999; COCHRANE; SCHULZE, 1999).

Os ambientes Ec e Pt, semelhantes entre si ( $\mathrm{p}=0,3337)$, encontram-se em áreas de tensão 
ecológica, onde há uma mescla de condições ambientais, com níveis de AD semelhantes a Cp (em torno de $80 \%$ ) e às unidades florestais (em torno de $25 \%$ ). Dada a heterogeneidade do dossel em Ec e ao crescimento de gramíneas em $\mathrm{Pt}$, há uma grande amplitude de variação entre os pontos amostrais, resultando em níveis intermediários de AD média para essas unidades.

Como esperado, $\mathrm{Cp}$ apresentou os maiores níveis de $\mathrm{AD}$, diferenciando-se das demais unidades $(\mathrm{p}<0,01)$. Essa unidade é dominada pelo estrato herbáceo, sem a formação de um dossel contínuo e, de acordo com as observações de campo, foi a unidade mais impactada pela queima. Isso pode estar relacionado à menor resistência desses ambientes à ocorrência de queimadas, pois o dossel aberto ou não formado deixa o ambiente mais inflamável, devido ao microclima mais favorável à queima e à maior quantidade de combustível (HENNENBERG et al., 2006; HOFFMANN et al., 2012). Da mesma forma, acredita-se que a baixa resistência à queima em formações campestres, sejam elas altimontanas, de Cerrado ou Campos sulinos, esteja relacionada à maior resiliência desses ambientes diante dos efeitos desse fenômeno, já que há maior ocorrência natural de fogo nesses ambientes, como citado por Oliveira-Filho e Fluminhan-Filho (1999) para o local deste estudo, e as espécies apresentam maiores adaptações para sobreviver aos seus efeitos (COUTINHO, 1990; OVERBECK et al., 2005; FIDELIS, 2008; SIMON et al., 2009).

\section{Conclusões}

De acordo com os resultados encontrados, as fitofisionomias semelhantes apresentam níveis de $\mathrm{AD}$ parecidos, da mesma forma que exibem um padrão de oscilação também similar entre si.

Quanto à avaliação do papel do fogo sobre o comportamento do dossel, a presente pesquisa conclui que o fogo foi um fator indireto de modificação da cobertura vegetal do parque, haja vista que a estrutura do dossel é capaz de controlar os impactos desse evento sobre a vegetação. Isto é, unidades com menor AD têm maior resistência aos efeitos das chamas, da mesma forma que o dossel mais aberto implica em unidades mais suscetíveis à queima e com maior capacidade de resiliência diante do fenômeno.

Além disso, destaca-se que a maior parte dos trabalhos encontrados é voltada para os efeitos do dossel sobre os estratos inferiores, diferente do que foi proposto nesta pesquisa, a qual trata da avaliação de fatores que modificam o dossel. Isso indica uma lacuna no conhecimento a respeito do tema, tornando necessária a replicação de estudos como este, para um melhor debate a respeito das informações levantadas neste trabalho.

\section{Referências}

ABRAMOFF, M. D.; MAGALHÃES, P. J.; RAM, S. J. Image processing with Image J. Biophotonics International, Pittsfield, v. 11, p. 36-42, 2004.

ALVARES, A. C. et al. Köppen's climate classification map for Brazil. Meteorologische Zeitschrift, Stuttgart, v. 22, n. 6, p. 711-728, 2013.

BIANCHINI, E.; PIMENTA, J. A.; SANTOS, F. A. M. Spatial and temporal variation in the canopy cover in a Tropical Semi-deciduous Forest. Brazilian Archives of Biology and Technology, Curitiba, v. 44, n. 3, p. 269-276, 2001.

BOND, W. J. What limits trees in C4 Grasslands and Savannas? Annual Review of Ecology, Evolution, and Systematics, Palo Alto, v. 39, n. 1, p. 641-659, 2008.

BOWMAN, D. M. J. S. et al. Fire in the Earth System. Science, Washington, v. 324, n. 5926, p. 
481-484, 2009.

BRASIL. Ministério da Agricultura e Reforma Agrária. Secretaria Nacional de Irrigação. Departamento Nacional de Meteorologia. Normais Climatológicas: 1961-1990. Brasília, 1992. 84 p.

BRASIL. Ministério do Meio Ambiente. Florestas do Brasil em resumo: dados de 2007-2012. Brasília: Serviço Florestal Brasileiro, 2013. 188 p.

COCHRANE, M. A. et al. Positive feedbacks in the fire dynamics of closed canopy Tropical Forests. Science, Washington, v. 284, p. 1832-1835, 1999.

COCHRANE, M. A. Fire Science for Rainforests. Nature, Londres, v. 421, n. 6926, p. 913-919, 2003.

COCHRANE, M. A.; SCHULZE, M. D. Fire as a recurrent event in tropical forests of the eastern Amazon: effects on forest structure, biomass, and species composition. Biotropica, Kansas, v. 31, n. 1, p. 2-16. 1999.

COUTINHO, L. M. Fire in the Ecology of Brazilian Cerrado. In: GOLDAMMER, J. G. Fire in the tropical biota: Ecological processes and global challenges. 1. ed. Berlim: Springer-Verlag, 1990. 497 p.

FENNER, M. The phenology of growth and reproduction in plants. Perpectives in Plant Ecology Evolution and Systematic, Massachusettes, v. 1, n. 1, p. 78-91, 1998.

FIDELIS, A. Fire in subtropical grasslands in Southern Brazil: effects on plant strategies and vegetation dynamics. 2008. Thesis (Doctorate in Science) - Technische Universität München, Munique, 2008.

FRAZER, G. W.; CANHAM, C. D.; LERTZMAN, K. P. Gap Light Analyzer (GLA), Version 2.0: Imaging software to extract canopy structure and gap light transmission indices from truecolour fisheye photographs, user's manual and program documentation. Burnaby: Simon Fraser University and Milbrook, Institute of Ecosystem Studies. 1999. 36 p.

GALINDO-LEAL, C.; CÂMARA, I. G. Mata Atlântica: biodiversidade, ameaças e perspectivas. São Paulo: Fundação SOS Mata Atlântica; Belo Horizonte: Conservação Internacional, 2005. 472 p.

GANDOLFI, S.; JOLY, C. A.; LEITÃO-FILHO, H. F. “Gaps of deciduousness”: cyclical gaps in tropical forests. Scientia Agricola, Piracicaba, v. 66, n. 2, p. 280-284, 2009.

GANDOLFI, S.; JOLY, C. A.; RODRIGUES, R. R. Permiability - Impermeability: canopy trees as biodiversity filters. Scientia Agricola, Piracicaba, v. 64, n. 4, p. 433-438, 2007.

HAINES, B.; FOSTER, R. B. Energy flow through litter in a Panamanian forest. Journal of Ecology, Oxford, v. 65, p. 147-155, 1977.

HENNENBERG, K. J. et al. Phytomass and fire occurrence along forest savanna transects in the Comoé National Park, Ivory Coast. Journal of Tropical Ecology, Cambridge, v. 22, n. 3, p. 303$311,2006$.

HOFFMANN, W. A. et al. Fuels or microclimate? Understanding the drivers of fire feedbacks at savanna-forest boundaries. Austral Ecology, Canberra, v. 37, n. 6, p. 634-643, 2012.

JOLY, C. A.; METZGER, J. P.; TABARELLI, M. Experiences from the Brazilian Atlantic Forest: ecological findings and conservation initiatives. New Phytologist, Lancaster, v. 204, n. 3, p. 459473, 2014.

JUDD, W. S. et al. Sistemática Vegetal: um enfoque filogenético. 3. ed. Porto Alegre: Artmed, 2009. $632 \mathrm{p}$. 
KLINK, C. A.; MACHADO, R. B. A conservação do Cerrado brasileiro. Megadiversidade, Rio de Janeiro, v. 1, n. 1, p. 147-155, 2005.

MAURO-DÍAZ, G.; LENCINAS, J. D.; DEL VALLE, H. Introducción a la fotografía hemisférica en ciencias forestales. Madera y Bosques, Veracruz, v. 20, n. 1, p. 109-117, 2014.

MICROSOFT. Microsoft Excel 2010. [S. l.], 2010.

MORELlAtO, L. P. C. et al. Phenology of Atlantic Rain Forest trees: a comparative study. Biotropica, Washington, v. 32, n. 4, p. 811-823, 2000.

MYERS, N. et al. Biodiversity hotspots for conservation priorities. Nature, London, v. 403, n. 6772, p. 853-858, 2000.

NICOTRA, B. A.; CHAZDON, R. L.; IRIARTE, S. V. B. Spatial heterogeneity of light and woody seedling regeneration in Tropical Wet Forests. Ecology, Washington, v. 80, n. 6, p. 1908-1926, 1999.

OLIVEIRA-FILHO, A. T.; FLUMINHAN-FILHO, M. Ecologia da vegetação do Parque Florestal Quedas do Rio Bonito. Cerne, Lavras, v. 5, n. 2, p. 51-64, 1999.

OVERBECK, G. E. et al. Fine-scale post-fire dynamics in Southern Brazilian subtropical grassland. Journal of Vegetation Science, Uppsala, v. 16, p. 655-664, 2005.

R CORE TEAM. R: a language and environment for statistical computing. Vienna: R Foundation for Statistical Computing, 2004. Disponível em: http://www.R-project.org/.

RICH, P. M. et al. Long-term study of solar radiation regimes in a tropical wet forest using quantum sensors and hemisferical photography. Agricultural and Forest Meteorology, Amsterdam, v. 65, p. 107-127, 1993.

SANTOS, N. D. et al. Bryophytic and phytogeographical aspects of two types of forest of the Serra do Mar State Park, Ubatuba/SP, Brazil. Biota Neotropica, Campinas, v. 11, n. 2, p. 425-438, 2011.

SIMON, M. F. et al. Recent assembly of the Cerrado, a neotropical plant diversity hotspot, by in situ evolution of adaptations to fire. Proceedings of the National Academy of Sciences, Washington, v. 106, n. 48, p. 20359-20364, 2009.

SINGH, K. P.; KUSHWAHA, C. P. Emerging paradigms of tree phenology in dry tropics. Current Science, Bangalore, v. 89, n. 6, p. 964-974, 2005.

SOUZA, F. M.; GANDOLFI, S.; RODRIGUES, R. R. Deciduousness influences the understory community in a Semideciduous Tropical Forest. Biotropica, Kansas, v. 46, n. 5, p. 512-515, 2014.

TABARELLI, M. et al. Prospects for biodiversity conservation in the Atlantic Forest: lessons from aging human-modified landscapes. Biological Conservation, Essex, v. 143, n. 10, p. 2328$2340,2010$.

TEIXEIRA, A. P.; ASSIS, M. A. Relação entre heterogeneidade ambiental e distribuição de espécies em uma floresta paludosa no Município de Cristais Paulista, SP, Brasil. Acta Botanica Brasilica, Belo Horizonte, v. 23, n. 3, p. 843-853, 2009. 